\title{
Infectious endophthalmitis: review of 420 cases
}

This article was published in the following Dove Press journal:

Clinical Ophthalmology

31 January 2013

Number of times this article has been viewed

Chavakij Bhoomibunchoo

Tanapat Ratanapakorn

Suthasinee Sinawat

Thuss Sanguansak

Kittipatra Moontawee

Yosanan Yospaiboon

Department of Ophthalmology, Faculty of Medicine, Khon Kaen University, Khon Kaen, Thailand
Correspondence: Yosanan Yospaiboon Department of Ophthalmology, Faculty of Medicine, Khon Kaen University, Khon Kaen, 40002, Thailand Tel +6643363010

Fax +66 43348383

Email yyosanan@gmail.com
Background: To characterize the causative pathogens and the visual outcomes among patients with endophthalmitis at a large referral center in northeastern Thailand.

Methods: All cases of infectious endophthalmitis treated between 1983 and 2007 were reviewed retrospectively. The patient data - including age, sex, history of ocular injuries, previous treatment and duration of the symptoms - were recorded. The ocular examination included affected side, anterior chamber reaction, vitreous haze, and presence of intraocular foreign bodies. Smears and cultures were prepared from anterior chamber paracentesis and/ or vitreous tapping. Intraocular antibiotics were administered at the time of anterior chamber paracentesis and/or vitreous tapping. Vitrectomy and/or lensectomy were performed when indicated. Initial visual acuity before treatment and the final best-corrected vision were compared.

Results: A total of 420 cases of endophthalmitis were reviewed: 181 cases (43.1\%) had ocular trauma before the infection; 135 (32.2\%) developed endophthalmitis after intraocular surgery; and, $122(29.1 \%)$ had a positive culture. Bacteria were isolated in 114 cases $(93.4 \%)$ and fungi were noted in eight (6.6\%). The common causative bacterium was Staphylococcus epidermidis. Combined vitrectomy and intraocular antibiotics were performed in 189 cases (45.0\%), whereas 69 cases $(16.4 \%)$ were treated with intraocular antibiotics alone.

Conclusion: Most of the reviewed cases were associated with trauma and intraocular surgery. The most frequently encountered bacterium causing infectious endophthalmitis was S. epidermidis. Most cases were treated with combined vitrectomy and intraocular antibiotics. The final visual outcomes seem to vary according to the type of endophthalmitis.

Keywords: endophthalmitis, visual outcome, Staphylococcus epidermidis

\section{Introduction}

Endophthalmitis may occur after a penetrating trauma, a recent intraocular surgery, an intravitreal injection, or originate from the bloodstream, usually by metastasis from distant foci of infection. ${ }^{1-8}$ In the past, endophthalmitis usually resulted in poor visual results or eye removal. ${ }^{9}$ However, with the use of effective intraocular antibiotics and advances in vitreoretinal surgery, the final visual outcome has markedly improved. ${ }^{1-4,10}$ There are few documented reports on the frequency of isolates of the specific causative microorganisms in endophthalmitis. A 25-year retrospective study of patients with endophthalmitis at a large referral center in northeastern Thailand was reviewed in order to characterize the spectrum of causative pathogens, the visual outcome, and identification of the factors affecting the prognosis. 


\section{Patients and methods}

Between 1983 and 2007, we studied all cases of suspected endophthalmitis treated at Srinagarind Hospital, Khon Kaen University, Thailand. This study followed the tenets of the Declaration of Helsinki and was approved by the Khon Kaen University Ethics Committee for Human Research. The patient data were recorded including: age, sex, occupation, history of ocular injuries, previous treatment, and duration of the symptoms. The ocular examination included the affected side, anterior chamber reaction, presence of hypopyon, vitreous haze, status of retina, and presence of intraocular foreign bodies. Smears and cultures were prepared from anterior chamber paracentesis and/or vitreous tapping. The smears were treated with Gram staining and potassium hydroxide $(\mathrm{KOH})$ wet preparation. Cultures were performed by plating on blood agar, chocolate agar, and McConkey agar incubated at $37^{\circ} \mathrm{C}$ for bacterial isolation. Sabouraud's dextrose agar without inhibitor and blood agar were incubated at $25^{\circ} \mathrm{C}$ to enhance fungal growth. Intraocular antibiotics were administered at the time of vitreous tapping and/or anterior chamber paracentesis as well as after vitrectomy or vitrectomy and lens removal. These antibiotics formerly included the combination of cefazolin and gentamicin or recently vancomycin and ceftazidime. If aspirate from vitrectomy was to be cultured, the material suctioned through the instrument was collected in a sterile bottle. The specimen was then centrifuged and the sediment used for smear and culture. In cases of painful blind eyes, enucleation was performed and the causative microorganisms could be isolated from the removed eyes. Snellen visual acuity was recorded in the affected eyes. Initial visual acuity before treatment and the final best-corrected vision were compared. One or more lines of improvement in the Snellen visual acuity chart was classified as "improved." After the 6/60 letter, 3/60, counting fingers, hand movement, light perception, and no light perception were considered the next line, respectively.

\section{Results}

A total of 420 cases of presumed endophthalmitis were included in this study. There were 283 male and 137 female patients. Age ranged from 1 month to 88 years with a median age of 45 years. Two hundred eighteen $(51.9 \%)$ left eyes and $198(47.2 \%)$ right eyes were affected. There were four cases $(0.9 \%)$ having both eyes affected. The duration of disease in most patients ranged between 1 day and 7 weeks (median, 3 days). Types of endophthalmitis in this study were demonstrated (Table 1). Episodes of ocular trauma occurred before the infection in 181 of the 420
Table I Types of endophthalmitis in patients

\begin{tabular}{ll}
\hline Types & Patients, n (\%) \\
\hline Exogenous & \\
Posttraumatic & $181(43.1 \%)$ \\
Penetration & $132(31.4 \%)$ \\
Intraocular foreign bodies & $40(9.5 \%)$ \\
Perforation & $9(2.2 \%)$ \\
Postoperative & $135(32.2 \%)$ \\
Cataract surgery & $117(27.9 \%)$ \\
Trabeculectomy & $7(1.7 \%)$ \\
Intravitreal injection & $6(1.4 \%)$ \\
Pars plana vitrectomy & $5(1.2 \%)$ \\
Postkeratitis & $13(3.1 \%)$ \\
Endogenous & $14(3.3 \%)$ \\
Undetermined & $77(18.3 \%)$ \\
\hline
\end{tabular}

patients (43.1\%). These included 132 cases (31.4\%) related to penetrating injury, 40 cases $(9.5 \%)$ with intraocular foreign bodies and nine cases associated with perforated wound. Endophthalmitis developed after intraocular surgery was noted in 135 patients $(32.2 \%)$. Our data also revealed that 13 cases (3.1\%) developed endophthalmitis after keratitis, 77 were undetermined (18.3\%), and 14 (3.3\%) with endogenous endophthalmitis were diagnosed by evidence of extraocular infection lesions. Most patients had poor initial visual acuities, and $336(80 \%)$ of them could only discern hand movement or less.

Smears and cultures were prepared from anterior chamber paracentesis and/or vitreous tapping. The results of positive smears and cultures from aqueous and vitreous tapping were compared (Table 2). Positive culture from either anterior chamber paracentesis or vitreous tapping or both were found in $122(29.1 \%)$ of 420 patients. Bacteria were isolated in 114 cases (93.4\%) and fungi in eight (6.6\%). They were Gram-positive in 64 cases (52.5\%), Gram-negative in 35 (28.7\%), and mixed organisms in $15(12.2 \%)$. The data on specific causative microorganisms are summarized and compared with other published reports in Table 3. The most frequent bacterium causing infectious endophthalmitis was Staphylococcus epidermidis, accounting for $17.54 \%$ of all bacterial cases. Other causative bacteria in order of frequency were Streptococcus pneumoniae (7.8\%), Pseudomonas aeruginosa (7.8\%), Bacillus spp. (7.0\%), Enterobacter spp. (6.1\%), Corynebacterium spp. (3.5\%), and Streptococcus spp. (2.6\%), respectively.

Table 2 Patients ( $\mathrm{n}$ [\%]) with positive smear and positive culture from aqueous and vitreous specimens

\begin{tabular}{llll}
\hline & Aqueous & Vitreous & Total \\
\hline Positive smear & $27(6.5 \%)$ & $88(20.9 \%)$ & $115(27.4 \%)$ \\
Positive culture & $12(2.9 \%)$ & $110(26.2 \%)$ & $122(29.1 \%)$ \\
\hline
\end{tabular}


Table 3 Causative agents (positive culture) in this study compared with previous studies

\begin{tabular}{|c|c|c|c|c|c|c|}
\hline Organisms & Peyman et al'" & Forster et $\mathrm{al}^{12}$ & Diamond $^{21}$ & Rowsey et $\mathrm{al}^{13}$ & Puliafito et al $^{14}$ & Present study \\
\hline \multicolumn{7}{|l|}{ Gram-positive } \\
\hline Staphylococcus epidermidis & 6 & 14 & 10 & 16 & 15 & 20 \\
\hline S. aureus & - & 7 & 4 & 8 & 2 & 3 \\
\hline Streptococcus pneumoniae & - & - & I & - & 2 & 9 \\
\hline Streptococcus spp. & 3 & 16 & 4 & - & 2 & 3 \\
\hline Bacillus spp. & - & - & 1 & 7 & I & 8 \\
\hline Corynebacterium & & & & & & 4 \\
\hline Enterococcus & & & & & & 3 \\
\hline Others & 3 & 8 & I & 7 & 2 & 14 \\
\hline \multicolumn{7}{|l|}{ Gram-negative } \\
\hline Pseudomonas aeruginosa & - & - & 1 & 2 & 1 & 9 \\
\hline Klebsiella spp. & & & & & & 2 \\
\hline Enterobacter spp. & - & - & - & 2 & I & 7 \\
\hline Proteous spp. & - & 12 & 1 & I & I & - \\
\hline Acinetobacter spp. & - & - & - & - & - & I \\
\hline Citrobacter spp. & & & & & & 2 \\
\hline Others & 3 & 11 & - & 3 & 2 & 14 \\
\hline Mixed bacteria & - & - & - & 5 & 6 & 15 \\
\hline \multicolumn{7}{|l|}{ Fungus } \\
\hline Fusarium solani & - & 3 & - & 2 & - & 2 \\
\hline Aspergillus spp. & - & 3 & - & 2 & - & I \\
\hline Candida albicans & 5 & 3 & 2 & - & - & 1 \\
\hline Curvularia & & & & & & 1 \\
\hline Others & - & 1 & 1 & - & 1 & 3 \\
\hline Total & 20 & 78 & 26 & 55 & 36 & 122 \\
\hline
\end{tabular}

All patients received topical and intravenous antibiotics. Drugs of choice included either cefazolin and gentamicin or vancomycin and amikacin or ceftazidime. Clindamycin was added in case of anaerobic infection. Combined vitrectomy and intraocular antibiotics were used on 189 cases (45.0\%), whereas $69(16.4 \%)$ were treated with intraocular antibiotics alone. Intraocular antibiotics included either cefazolin and gentamicin or vancomycin and ceftazidime. Enucleation was performed in 75 cases (17.9\%), three of which had their eyes removed in a second operation. Conservative therapy, defined as appropriate topical, periocular, and systemic antibiotics, was used in 87 cases $(20.7 \%)$.

The initial visual acuity and final visual outcome were compared. The visual acuities were improved in 253 cases $(60.2 \%)$, stable in $58(13.8 \%)$, and worse in $109(26 \%)$. The visual outcomes according to the types of endophthalmitis are presented in Figures 1 and 2.

\section{Discussion}

Most cases in our study were associated with ocular trauma, which is dissimilar to previous reports in which most cases developed endophthalmitis after intraocular surgery., ${ }^{9,1-15}$ The discrepancy might be attributed to the higher incidence of ocular injury with vegetative matter in an agrarian culture as is found in northeastern Thailand. Early diagnosis and prompt treatment are the two most important steps in the management of patients with suspected endophthalmitis. Diagnosis should be accomplished by clinical and laboratory studies, including anterior chamber paracentesis and vitreous tapping. Microbiological investigation of intraocular fluid is the only method that permits reliable identification of the causative microorganisms, and intraocular fluid culture is still used as a gold standard for diagnosis. Vitreous fluid specimens in blood culture bottles have been reported to yield more positive results than conventional culture media and improve the chance of successful treatment. ${ }^{16,17}$ However, prompt antimicrobial therapy should depend primarily on the initial smear of the specimens obtained, not the culture results. This is because by the time the organism is identified through culture and the antibiotic sensitivity determined (24-36 hours), serious further visual loss and ocular damage may have occurred. Thus, broad-spectrum intraocular bactericidal antibiotics should be given immediately after examination of the aqueous and vitreous specimens using Gram's stain and $\mathrm{KOH}$ wet smear. Later, after the organism has been identified, the antimicrobial therapy can be made more specific, according to the sensitivity data and clinical response. 


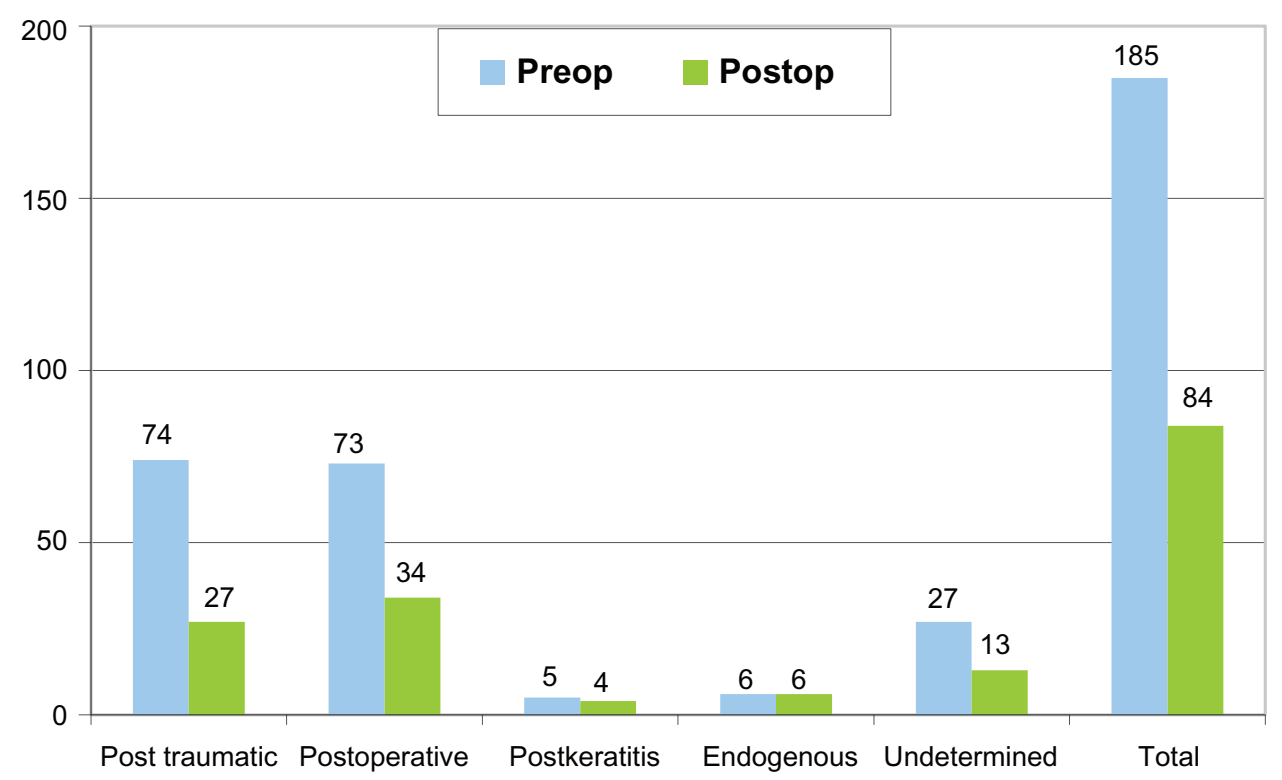

Figure I Demonstrated decreasing number of patients who had visual acuity worse than hand motion (pre- vs postoperation).

In our study, the most frequent bacterium causing infectious endophthalmitis was $S$. epidermidis. This result agrees well with other previous studies. ${ }^{11-15}$ In the current study, it was also noted that Fusarium solani was the most frequent fungal isolate in eyes with endophthalmitis, which agrees with reports from other tropical countries, but not with North American studies. ${ }^{18,19}$ In previous reports, all Bacillus endophthalmitis occurred after a penetrating injury and were associated with an intraocular foreign body, ${ }^{1,2}$ but it was not the case in our study. Two cases with Bacillus infection in this report had no intraocular foreign body although both occurred after a penetrating trauma caused by vegetative matter. A poor visual prognosis in Bacillus endophthalmitis was noted in all studies including ours. In our study, two patients infected by this virulent organism required enucleation, while others treated with a combination of vitrectomy and intraocular antibiotics had a poor visual result even though the eye was saved.

There are various recommendations for the treatment of endophthalmitis. In cases with less virulent organisms, treatment without intraocular administration of antibiotics may be successful. O'Day et al reported satisfactory

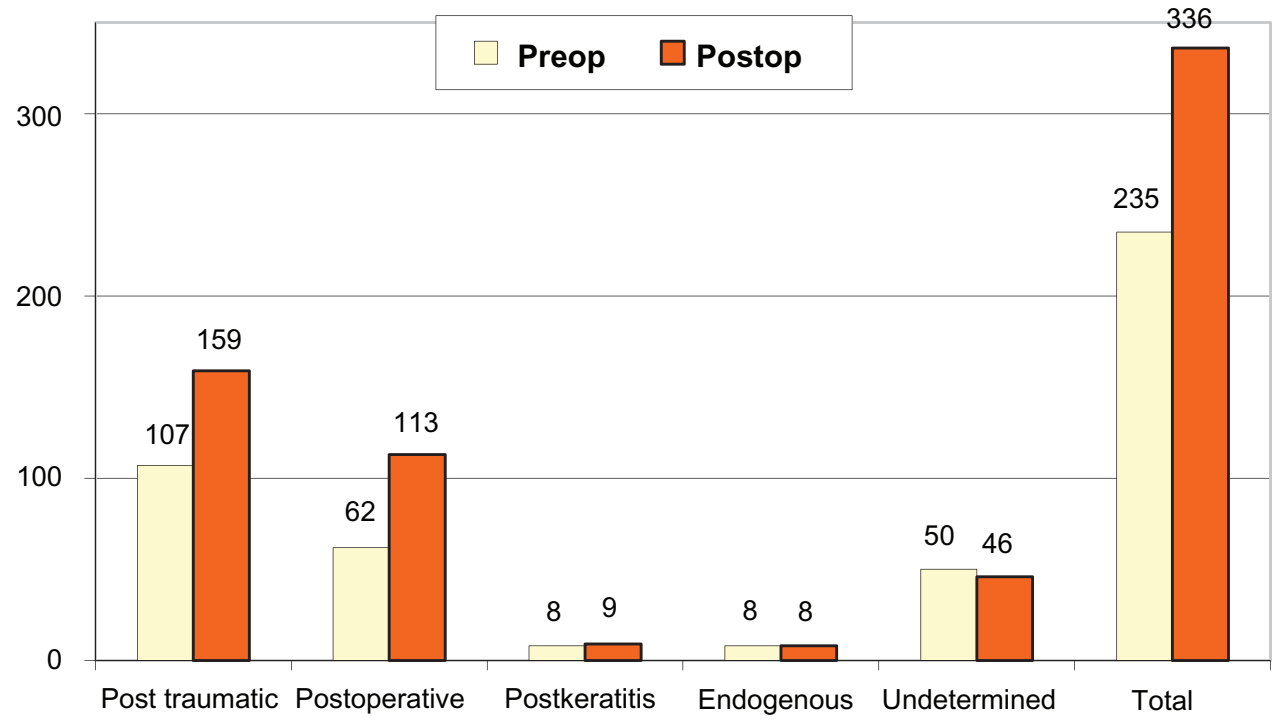

Figure 2 Demonstrated increasing number of patients who had visual acuity better than hand motion (pre- vs postoperation). 
results in treating $S$. epidermidis endophthalmitis with conventional therapy (ie, topical, subconjunctival and systemic antibiotics). ${ }^{20}$

Laboratory studies and clinical evidences have, however, also demonstrated relatively superior results with intravitreal antibiotics over conventional routes. Forster et al reported a final visual acuity of $20 / 400$ in 8 of $14(57 \%)$ culturepositive eyes treated with intraocular antibiotics alone. ${ }^{12}$ Diamond also reported a final visual acuity of 20/400 or better in 10 of $12(83 \%)$ ) eyes treated with intravitreal and oral antibiotics. ${ }^{21}$ Although the clinical data did not permit a valid comparison between intraocular and conventional routes, it appears from pharmacological studies that direct intraocular injection of antibiotics provides drug levels superior to topical, periocular, or systemic application. ${ }^{22}$ However, many studies show bactericidal intraocular levels of systemically given cephalosporins and other antibiotics when measured in inflammatory conditions where the blood-eye barriers do not exist. ${ }^{20}$ Since a definite causative organism cannot be identified at the time of paracentesis and vitreous aspiration until the culture and sensitivity results are reported, the risk/ benefit ratio is strongly in favor of intravitreal injection in virtually every instance of infectious endophthalmitis.

Despite advances in the diagnosis and treatment of infectious endophthalmitis, the eventual visual result in these patients remains unsatisfactory. A review of all reported cases of endophthalmitis between 1950 and 1977 showed that $67 \%$ resulted in no light perception or removal of the affected eye(s). ${ }^{9}$ Since 1980, the success rate - defined as final vision of 20/400 or better - has increasingly improved, ranging from $42 \%$ to $73 \% .^{1-4,10}$ In the current study, only $17.9 \%$ of the patients had no light perception or underwent removal of the affected eye probably because of the advent of vitreoretinal microsurgery at our hospital since 1985. Vitrectomy also has satisfactory results in the management of this severe infection. This surgical procedure can serve as a therapeutic incision for drainage; removing the bulk of infectious organisms and associated inflammatory debris from the eye, providing a large quantity of vitreous for diagnostic smear and culture, and permitting better antibiotics distribution. ${ }^{23}$ In a rabbit model of endophthalmitis caused by intravitreal injection of Bacillus cereus, Callegan et al demonstrated that eyes treated by combined vitrectomy and intraocular vancomycin during the early stage of infection resulted in significantly greater retinal function compared with that of intraocular antibiotics alone. ${ }^{24}$ In the current study, 189 eyes with endophthalmitis were treated with a combination of vitrectomy and intraocular antibiotics. Although the evaluation of the effect of this surgical procedure cannot be established due to a lack of a valid comparison, vitrectomy should be considered in certain severe cases that have shown to be resistant (or not responsive) to the medical therapy. Moreover, the technological advances of small-gauge vitrectomy seem to afford visual benefit, with more rapid healing, less discomfort, and an acceptably low incidence of adverse events compared with those observed in conventional 20 -gauge vitrectomy. ${ }^{25}$ The final visual outcomes in the current study showed various results in each group of patients; all groups showed a reduction in the number of the patients who had poor visual acuity (less than hand motion) except in the endogenous endophthalmitis group.

\section{Conclusion}

A retrospective review of 420 cases of infectious endophthalmitis was conducted. Most cases were associated with trauma and postoperative intraocular surgery, respectively. The common causative bacteria in order of reducing frequency were $S$. epidermidis, $S$. pneumoniae, P. aeruginosa, Bacillus spp., Enterobacter spp., Corynebacterium spp., and Streptococcus spp. Despite aggressive management for our patients, about one-fourth still had a poor final visual outcome. Early detection and prompt management seem to be the most important factors for improving final visual outcomes. Although this study is limited by its retrospective nature and restriction on specific information and some essential data, it is a large endophthalmitis study and presents additional data on endophthalmitis to the literature. Further prospective study should be designed to meet the specific needed information.

\section{Acknowledgments}

This study was supported by an invitation research grant (I-50138) from the Faculty of Medicine, Khon Kaen University, Khon Kaen, Thailand. The authors thank the Department of Ophthalmology and the Faculty of Medicine, Khon Kaen University for support and Mr Bryan Roderick Hamman and Mrs Janice Loewen-Hamman for assistance with the English-language presentation of the manuscript.

\section{Disclosure}

The authors report no proprietary or conflicts of interest in this work.

\section{References}

1. Bhagat N, Nagori S, Zarbin M. Post-traumatic infectious endophthalmitis. Surv Ophthalmol. 2011;56(3):214-251. 
2. Ahmed Y, Schimel AM, Pathengay A, Colyer MH, Flynn HW Jr. Endophthalmitis following open-globe injuries. Eye. 2012;26(2): 212-217.

3. Zhang Y, Zhang MN, Jiang CH, Yao Y, Zhang K. Endophthalmitis following open globe injury. Br J Ophthalmol. 2010;94(1):111-114.

4. Sheng Y, Sun W, Gu Y, Lou J, Liu W. Endophthalmitis after cataract surgery in China, 1995-2009. J Cataract Refract Surg. 2011;37(9):1715-1722.

5. Pathengay A, Flynn HW Jr, Isom RF, Miller D. Endophthalmitis outbreaks following cataract surgery: causative organisms, etiologies, and visual acuity outcomes. J Cataract Refract Surg. 2012;38(7): 1278-1282.

6. Moshfeghi AA. Endophthalmitis following intravitreal anti-vascular endothelial growth factor injections for neovascular age-related macular degeneration. Semin Ophthalmol. 2011;26(3):139-148.

7. Sadaka A, Durand ML, Gilmore MS. Bacterial endophthalmitis in the age of outpatient intravitreal therapies and cataract surgeries: host-microbe interactions in intraocular infection. Prog Retin Eye Res. 2012;31(4):316-331.

8. Riddell J 4th, Comer GM, Kauffman CA. Treatment of endogenous fungal endophthalmitis: focus on new antifungal agents. Clin Infect Dis. 2011;52(5):648-653.

9. Micheal SK, Alessandro AC, Marco AZ. Endophthalmitis. Surv Ophthalmol. 1998;43:195-224.

10. Jonathon QN, Nigel M, John WP, et al. Management and outcomes of postoperative endophthalmitis since the Endophthalmitis Vitrectomy Study. The Endophthalmitis Population Study of Western Australia (EPSWA)'s Fifth Report. Ophthalmology. 2005;112:1199-1206.

11. Peyman GA, Richand M, Bennett TO. Management of endophthalmitis with pars plana vitrectomy. Br J Ophthalmol. 1980;64:472-475.

12. Forster RK, Abbott RL, Celenger H. Management of infectious endophthalmitis. Ophthalmology. 1980;87:313-318.
13. Rowsey JJ, Newsom DL, Sexton DJ, et al. Endophthalmitis: current approaches. Ophthalmology. 1982;89:1055-1065.

14. Puliafito CA, Baker AS, Haat J, et al. Infectious endophthalmitis: review of 36 cases. Ophthalmology. 1982;89:921-929.

15. Humeyra K, Steven MK, Rajendra SA. Retrospective analysis of etiopathogenesis of all cases of endophthalmitis at a large tertiary referral center. Int Ophthalmol. 2007;27:251-259.

16. Ness T, Pelz K. Endophthalmitis: improvement of culture results. Ophthalmology. 2000;97:33-37.

17. Yospaiboon Y, Saree S, Pasadhika S. Blood culture and conventional culture media for vitreous culture in endophthalmitis. J Med Assoc Thai. 2005;88(5):639-642.

18. Yospaiboon Y, Sangvijit J, Suwanwantana C. Endophthalmitis: review of 77 consecutive cases. Thai J Ophthalmol. 1988;2:63-69.

19. Chhablani J. Fungal endophthalmitis. Expert Rev Anti Infect Ther. 2011;9(12):1191-1201.

20. O'Day DM, Jones DB, Patrineli J, et al. Staphylococcus epidermidis endophthalmitis: visual outcome following non-invasive therapy. Ophthalmology. 1982;89:354-360.

21. Diamond JG. Intraocular management of endophthalmitis: a systemic approach. Arch Ophthalmol. 1981;99:96-99.

22. López-Cabezas C, Muner DS, Massa MR, Mensa Pueyo JM. Antibiotics in endophthalmitis: microbiological and pharmacokinetic considerations. Curr Clin Pharmacol. 2010;5(1):47-54.

23. Maguire JI. Postoperative endophthalmitis: optimal management and the role and timing of vitrectomy surgery. Eye. 2008;22(10):1290-1300.

24. Callegan MC, Guess S, Wheatley NR, et al. Efficacy of vitrectomy in improving the outcome of Bacillus cereus endophthalmitis. Retina. 2011;31(8):1518-1524.

25. Recchia FM, Scott IU, Brown GC, Brown MM, Ho AC, Ip MS. Small-gauge pars plana vitrectomy: a report by the American Academy of Ophthalmology. Ophthalmology. 2010;117(9):1851-1857.
Clinical Ophthalmology

\section{Publish your work in this journal}

Clinical Ophthalmology is an international, peer-reviewed journal covering all subspecialties within ophthalmology. Key topics include: Optometry; Visual science; Pharmacology and drug therapy in eye diseases; Basic Sciences; Primary and Secondary eye care; Patient Safety and Quality of Care Improvements. This journal is indexed on Submit your manuscript here: http://www.dovepress.com/clinical-ophthalmology-journal

\section{Dovepress}

PubMed Central and CAS, and is the official journal of The Society of Clinical Ophthalmology (SCO). The manuscript management system is completely online and includes a very quick and fair peer-review system, which is all easy to use. Visit http://www.dovepress.com/ testimonials.php to read real quotes from published authors. 